\title{
Formation and evolution of dust in novae
}

\author{
A. Evans \\ Department of Physics, University of Keele, Keele, Staffordshire, ST5 5BG, United Kingdom.
}

\begin{abstract}
The formation and evolution of dust grains following a nova outburst are reviewed, and various observational methods of constraining the nature of nova dust are described. Despite recent advances in understanding grain formation and evolution in classical nova systems, there remains a number of outstanding problems, the solutions to which are likely to come from studies of other aspects of the nova outburst.
\end{abstract}

\section{Introduction}

The idea that dust might form in the ejecta of classical novae was first mentioned by McLaughlin (1935), in an attempt to understand the spectacular deep minimum that occurred in the visual light curve of DQ Her. Thereafter the subject seems to have gone into hibernation, until the discovery by Geisel et al. (1970) of the infrared excess of FH Ser. During the 1980's there were a number of reviews, both of the infrared development of novae and of their dust formation properties (Bode \& Evans 1983b, 1989; Gehrz 1988, 1989), and accordingly we will concentrate here on material reported since these reviews.

The topic of dust formation and evolution in novae may conveniently be divided into three general areas, namely (i) nucleation, (ii) evolution in the nova environment, and (iii) post-nova history. We shall discuss each of these topics in turn but first we review briefly what is known about the nature of nova dust.

\section{The composition of nova dust}

Information about the composition of nova dust can be provided in several ways, namely (i) the infrared flux distribution; (ii) abundances, both as predicted by thermonuclear runaway (TNR) models and as determined observationally; (iii) the ultraviolet-optical extinction law; (iv) observed condensation temperatures.

\subsection{Infrared flux distribution}

Early observational work on nova dust shells consisted almost entirely of broad-band photometry but even so this was generally sufficient to show that the flux distribution was relatively smooth (see Gehrz 1988, Bode \& Evans 1989 and references therein). This property, coupled with the knowledge that the CNO group of elements is overabundant in nova ejecta, led to the general assumption that the condensate is likely to be carbon.

However in recent years it has become apparent that the flux distribution of the dust emission of some novae is not always featureless. In a number of cases (see Gehrz 1988, 1989 for details) there is a prominent feature in the $8-13 \mu \mathrm{m}$ window which has been attributed both to silicates (which show a peak at $9.7 \mu \mathrm{m}$ ) and to silicon carbide (which has a peak at $11.5 \mu \mathrm{m}$ ). Indeed the possibility of a feature at $11.5 \mu \mathrm{m}$ in the dust emission of NQ Vul was suggested by Mitchell et al. (1983) on the basis of broad-band photometry alone. In the case of V1370 Aql the $10 \mu \mathrm{m}$ feature was attributed by Bode et al. (1984) and Roche et al. (1984) to silicates. The problem with the silicate interpretation in this case is the absence of a $20 \mu \mathrm{m}$ feature and this led Gehrz et al. (1984) to tentatively identify the condensate as $\mathrm{SiC}$. The $10 \mu \mathrm{m}$ feature results from $\mathrm{Si}-\mathrm{O}$ 
stretch while the $20 \mu \mathrm{m}$ feature arises from $\mathrm{O}-\mathrm{Si}-\mathrm{O}$ bend. The absence of the $20 \mu \mathrm{m}$ feature might therefore be consistent with a material (like $\mathrm{SiO}$ ) that contains the former bond but not the latter; however, for reasons outlined by Gehrz et al. (1984), the SiO identification is unlikely. Another alternative $\left(\alpha+\beta \mathrm{Si}_{3} \mathrm{~N}_{4}\right)$ has been suggested by Goebel (1986) on the basis of spectral comparison with laboratory data; this produces a good spectral fit around $10 \mu \mathrm{m}$ without giving rise to features at other wavelengths.

Emission features in the $3 \mu \mathrm{m}$ window might be expected if nova dust is carbon-based. These have now been observed and are discussed in Section 4.3 below.

\subsection{Abundances}

The predicted and observed abundances of species that are likely to condense in nova winds are valuable pointers to the composition of nova dust. The canonical view is that silicates will condense in a stellar outflow in which $\mathrm{O}$ is more abundant than $\mathrm{C}$ by number, otherwise carbon or silicon carbide will condense. However recent novae have confounded this simple-minded view by apparently forming both ' $\mathrm{C}$-rich' and ' $\mathrm{O}$-rich' dusts.

The fact that carbon seems to be a condensate in at least some novae indicates that $\mathrm{C}$ must be overabundant relative to $O$. In general it seems that TNR models invariably predict that $O$ is the more abundant species; however the formation of carbon dust and its implication for the $C / O$ ratio is an unavoidable observational constraint that has to be addressed by TNR models.

Abundances may be determined observationally but this is generally possibly only during the nebular phase, after any grain formation has taken place, so that condensing species are significantly depleted. A list of abundances in nova ejecta, as determined from $I U E$ data, is given by Snijders et al. (1984). An excellent, and so far the only, example of the relation between ejecta abundances and grain composition is provided by the IUE observations of V1370 Aql, in which the silicon abundance was seen to decline as grains condensed (Snijders et al. 1986).

\subsection{Circumstellar extinction}

In principle the circumstellar extinction law can-via Mie theory-provide information not only about the nature of the dust but also about grain size and possibly even grain shape. A closely related phenomenon is the wavelength- and time-dependence of optical-infrared polarization, which can also pin down grain size and shape.

In practice it is rather difficult to determine the circumstellar extinction laws for novae for several reasons. First, simultaneous observations in two or more wavebands are ideally required; second, accurate knowledge of the intrinsic flux distribution is necessary; third, the interstellar extinction law in the direction of the nova must be known (simply taking an 'average' extinction law is unlikely to be adequate); and finally, in comparing an observed extinction law with Mie theory one has to make assumptions about the geometry of the nova dust shell so that scattering can properly be taken into account.

Despite these difficulties Snijders et al. (1986) have used IUE spectra in an attempt to determine the circumstellar extinction law for V1370 Aql. They found that the extinction law for the nova dust had a peak at wavelength $2500 \AA$-compared with the peak in the interstellar extinction law at $\sim 2175 \AA$-which they interpreted in terms of small $(0.013 \mu \mathrm{m})$ carbon particles. The difficulty with this interpretation is that the grain size distribution has to be sharply peaked around this value otherwise the extinction peak is smeared out. Nonetheless the work of Snijders et al. does demonstrate that the extinction law for nova dust is accessible provided the above difficulties can be overcome. 


\subsection{Condensation temperatures}

The chemical composition of the dust (amongst other factors) has significant bearing on the temperature at which condensation occurs and the condensation temperature is a quantity that can in principle be determined from broad-band photometry. In practice however the determination of the condensation temperature is not straightforward because of the generally inadequate time resolution of the observations. The best that can be done is to determine the dependence of dust temperature on time and then extrapolate back to the expected condensation time (Bode \& Evans 1983a), although this procedure has the drawback of being model-dependent.

Bode \& Evans (1983a) attempted to determine condensation temperatures in this way for a number of novae for which there existed adequate infrared photometry. They found condensation temperatures in the range 1200-2000 K, the upper end of the range corresponding to the expected condensation temperature of carbon in nova environments. In general though this approach has not been too helpful in identifying the nature of the condensate, although Lewis \& Ney (1979) suggested that condensation temperatures in novae (and other objects) pointed to iron or cohenite $\left(\mathrm{Fe}_{3} \mathrm{C}\right)$ as a condensate.

\subsection{Summary}

The comment has been made (Bode \& Evans 1989) that the nature of the dust that condenses in nova winds may depend on the nature of the white dwarf: a nova binary in which there is a CO white dwarf will (if it produces dust at all) produce carbon dust, whereas a nova binary with a $\mathrm{MgNeO}$ white dwarf (which produces a 'neon' nova) will produce silicate dust. [This also implies that some of the white dwarf is carried up into the accreted envelope and ejected in the course of the outburst.] However in view of the variety of dust types produced in some individual novae (see below) even this generalization probably does not get anywhere near the truth.

Whatever determines the nature of nova dust the problem of nucleation is easier to address if the dust is composed of a simple material like carbon, even if only for the fact that we know that the eventual condensate exists in the gas phase; the growth of (say) $\mathrm{MgSiO}_{3}$ from the gas phase must presumably take place via some indirect route, probably chemical reactions on grain surfaces, and the problem of nucleation is not straight-forward. There is the also the intermediate case of silicon carbide: the presence of the $\mathrm{SiC}$ molecule in the gas phase is well-known in both circumstellar and interstellar environments and recent laboratory work (see below) suggests that $\mathrm{SiC}$ may well nucleate homogeneously.

\section{Grain nucleation}

The problem of grain nucleation in novae, in common with grain nucleation in many other astrophysical environments, poses many problems, although these are exacerbated in the nova context because of the harsh environment in which the grains have to form. However what theoreticians find very difficult to understand, Nature seems to achieve with little difficulty. There is no difficulty in getting the grains to grow once nucleation has occurred: it is the initial nucleation process that poses problems.

In novae we seem to have a classic 'Catch 22' situation. We start off with atoms and ions and need to end up with grains; there must be an intermediate phase where molecules are produced. As is well known (Duley \& Williams 1984) the $\mathrm{H}_{2}$ molecule drives most of the chemistry in the interstellar medium, where $\mathrm{H}_{2}$ molecules form on grain surfaces. In novae, we again need $\mathrm{H}_{2}$ to drive the chemistry but apparently $\mathrm{H}_{2}$ can not form without grains, while grains can not form before $\mathrm{H}_{2}$ molecules exist to drive the chemistry... 
However recent work, both theoretical (on the chemistry of nucleation) and experimental, suggests that these difficulties are not insurmountable. One can attack the problem of grain nucleation in nova ejecta from two points of view. First we can try to understand how nucleation centres can form in the ejected material and second, we can investigate whether suitable nucleation centres are already present in the pre-outburst environment. We discuss each of these in turn.

\subsection{Nucleation in the ejecta}

In order to make any headway as far as nucleation in the ejected material is concerned we must get some sort of estimate of the nucleation rate, i.e. the number of nucleation centres produced per unit volume per unit time. We can do this by looking at a typical dusty nova, which produces $\sim 10^{39}$ grains in about 10 days, in a volume $\sim 10^{45} \mathrm{~cm}^{3}$; the resulting nucleation rate $J \sim 10^{-12} \mathrm{~cm}^{-3} \mathrm{~s}^{-1}$. Any nucleation process must be capable of meeting this observational constraint. Although nova dust is mineralogically rather diverse, we shall consider the nucleation problem mainly in the context of carbon as this is the most straightforward case.

Nucleation can be either homogeneous or heterogeneous (see e.g. Draine 1979 for a review). It has been apparent for some time that the formation of carbon dust in novae cannot occur via homogeneous nucleation. From expressions in Donn et al. (1968), and taking values typical of nova ejecta at the time of grain formation, we get predicted nucleation rates $\sim 10^{-25} \mathrm{~cm}^{-3} \mathrm{~s}^{-1}$, well below that required. The main problem here is the ' $\mathrm{C}_{2}$ bottleneck', the rate for the initial reaction $\mathrm{C}+\mathrm{C} \rightarrow \mathrm{C}_{2}+h \nu$ being $\sim 10^{-17} \mathrm{~cm}^{3} \mathrm{~s}^{-1}$ (Duley \& Williams 1984). We must therefore rely on heterogeneous nucleation to form carbon dust in novae.

Gallagher (1977) suggested that the state of ionization (of hydrogen) in nova ejecta might inhibit nucleation and grain formation by virtue of coulomb repulsion. However as pointed out by Mitchell \& Evans (1984) it is the state of ionization of the potential condensate (in this case carbon) rather than that of hydrogen which is relevant. As the ejecta expand away from the nova they acquire a complex ionization structure, species having low ionization potential (like $\mathrm{Fe}$ and $\mathrm{Mg}$ ) are completely ionized virtually instantaneously, whereas the ionization time for carbon is a few days; further, the carbon ionization front is extended. There is thus a brief interval, of a few days' duration, when neutral carbon co-exists with a variety of ions. Callus et al. (1987) therefore suggested that nucleation of carbon might occur on ions, because reactions of the form $\mathrm{X}^{+}+\mathrm{C} \rightarrow \mathrm{CX}^{+}+h \nu$, where $\mathrm{X}^{+}$is a metallic ion, have more favourable rates than $\mathrm{C}+\mathrm{C}$ (e.g. Duley \& Williams 1984). However, as discussed by Rawlings \& Williams (1989), the reaction products in this case are vulnerable to dissociation in the harsh radiation field and for this reason ion nucleation is unlikely to be important.

An alternative possibility is that grains may nucleate on molecules. It is well known that, even in dustless novae such as V1500 Cyg, a prominent feature appears around $5 \mu \mathrm{m}$ during the free-free phase. Although much of the early observational data were broadband it seems certain that this feature is the fundamental vibration-rotation transition of $\mathrm{CO}$ at $4.8 \mu \mathrm{m}$. Rawlings (1988) has shown that $\mathrm{CO}$ can indeed form in nova winds in a thin, dense, cool region at the outer edge of the ejecta. As already noted, a prerequisite for $\mathrm{CO}$ formation is the formation of $\mathrm{H}_{2}$, the function of which is to initiate the chemistry and to shield the outer regions of the ejecta from the radiation emitted by the stellar remnant; nova winds are (unlike the interstellar medium) dense enough for $\mathrm{H}_{2}$ to form via 3-body reactions. As is well known, when dust formation does occur the $4.8 \mu \mathrm{m}$ $\mathrm{CO}$ feature disappears, either as a result of its being subsumed in the grain formation process or (more likely) as a result of its destruction in the nova environment.

Following Rawlings' (1988) initial investigation into the formation of CO in nova winds, Rawlings \& Williams (1989) have investigated in detail the chemical routes to the formation of nucleation sites in nova winds. A prerequisite, as indeed it is for the formation of $\mathrm{CO}$, is that there 
exists in the ejected material a region in which the atomic carbon is neutral. As shown by Mitchell \& Evans (1984) and Rawlings (1988), the ionization time for carbon is only a few days so the time available for the formation of nucleation sites is very short indeed. Rawlings \& Williams stress that, contrary to previous discussions in the context of novae, grain formation must occur in a situation that is extremely non-LTE: the kinetic temperature where nucleation is occurring is $1000-2000 \mathrm{~K}$, but the radiation field corresponds to blackbody emission at several times $10^{4} \mathrm{~K}$. As a consequence of the latter the lifetimes of some crucial species in the nucleation reaction network is extremely short ( $\sim$ seconds) and unless the region of nucleation is adequately shielded the formation of nucleation sites must occur on this sort of timescale. Once carbon is ionized, $\mathbf{H}_{2}$ is destroyed and molecule formation is inhibited by the radiation field.

However in the few days while the carbon is neutral the outer regions of the ejecta are shielded from the stellar remnant and chemistry can proceed. During this phase hydrocarbon molecules are formed and these provide the required nucleation sites for the formation of carbon dust. Rawlings \& Williams (1989) argue that the formation of CO is a necessary (but not sufficient) condition for hydrocarbon formation: $\mathrm{CO}$ serves to deplete the oxygen so that the hydrocarbon nucleation sites are not 'burned'. The authors find that the number of nucleation sites formed is consistent with the observed number of grains produced by novae (see above) provided the grains are large $(\sim \mu \mathrm{m})$ rather than small, as indeed was demonstrated by the detailed investigation of the infrared development of NQ Vul by Mitchell et al. (1983).

Johnson et al. (1987) have also looked at chemical routes of grain nucleation in carbon-rich novae and have developed a kinetic approach to grain nucleation. They find an embarrassment of nucleation centres and, as discussed by (for example) Draine (1979), too many nucleation centres results in very small grains. However the form of the flux distribution demands $\mu \mathrm{m}$-sized grains and a means is required of inhibiting the nucleation process to cut down on the number of nucleation sites. Johnson et al. invoke temperature fluctuations and evaporation of nucleation sites (induced by stochastic heating) to achieve this.

Another route for heterogeneous nucleation has been prompted by recent experimental work on the homogeneous nucleation of silicon carbide (Carmer \& Frenklach 1989). Although the possibility of silicon carbide dust in novae is a contentious issue (see Gehrz 1988) a number of 'neon' novae do show a prominent feature in the 8-13 $\mu \mathrm{m}$ window. Frenklach et al. (1989) show that, in a carbon rich outflow, the first condensate will be silicon carbide, which nucleates homogeneously. Silicon carbide particles, of $\sim 100 \AA$ dimension, then act as nucleation centres for the growth of carbon grains. One interesting possibility here is that, if carbon is depleted by the formation of silicon carbide to the extent that $\mathrm{C}$ becomes numerically underabundant relative to $\mathrm{O}$, the grains that subsequently condense may be silicate, so that we end up with silicate mantles on a silicon carbide core. This may have some bearing on the 'mysterious' silicate feature seen in some novae (e.g Gehrz et al. 1984).

An intriguing possibility is that carbon may condense in the winds of some novae in the form of the 'soccer-ball' molecule buckminsterfullerene, $C_{60}$ (e.g. Curl \& Smalley 1988; Kroto 1988). Kroto suggests that carbon clusters with 30-100 atoms (e.g. corannulene) are formed as icospirals of dimensions $\sim 10 \AA$ in the process of carbon nucleation. As growth proceeds closure is possible resulting in $\mathrm{C}_{60}$. In a nova this provides an ideal means of locking up radioactive species (like ${ }^{22} \mathrm{Na}$ ), which become 'caged' in the hollow $\mathrm{C}_{60}$ molecule ('metallofullerenes'); this could be relevant in the context of laboratory investigations of meteorites (see below). Another possibility is that, instead of closure, the fullerene molecule may have hydrogen atoms at vertices which would normally be occupied by carbons ('hydrofullerene'); this again could be relevant in the context of $3 \mu \mathrm{m}$ emission features in nova dust shells (see below). 


\subsection{Pre-existing nucleation sites}

Although we are now working towards an understanding of nucleation of nova dust, the difficulty of getting nucleation going in nova winds has prompted many to suppose that the required nucleation sites might pre-date the nova outburst, so that growth can proceed once the ejected material reaches the condensation nuclei. Note that this is only moving the question back a stage: one still has to consider the origin of the pre-existing nucleation sites. There are at least two possibilities, which we now discuss.

Jenkins \& Evans (1988) have shown that the existence of grains in the accretion disks of cataclysmic variables is a distinct possibility. In the case of nova binaries having main sequence secondaries the situation is marginal, the existence of accretion disk grains depending on the mass transfer rate, viscosity parameter and temperature gradients in the disk, abundance anomalies in the secondary etc. The situation is much more favourable in the case of novae and nova-like systems having giant secondaries (including recurrent novae and symbiotics). In either case the grains might form either in the accretion disk itself, or may be carried over from the atmosphere of the secondary in the inter-star stream (the transit time of these grains in the 'hot-spot' is not long enough to cause serious damage). The radiation pressure from the disk, particularly from the hotter inner regions, is then generally sufficient to blow the grains out. Clearly the process can not occur during 'hibernation' (e.g Shara et al. 1986).

Another means of producing nucleation sites has been suggested by Matese et al. (1989). These authors note that, if a nova binary has an Oort-type cometary cloud, any volatiles will be sublimed at the time of eruption, releasing dust into the circumstellar environment. Such material might then emit infrared radiation as a result of the 'infrared echo' (e.g. Bode \& Evans 1980), giving rise to a weak infrared excess as seen in the nova PW Vul (Gehrz et al. 1988). Indeed, as noted by Gehrz (1988), the behaviour of the infrared excess of novae having weak excesses is not inconsistent with the infrared echo model. If, on the other hand, the ejected material encounters the cometary dust and condensation is kinetically favourable, grain growth (and a strong infrared excess) will ensue. However this model, in which the dust is confined to a disk, would not account for the infrared development of moderate speed novae having strong infrared excess. In these objects the evidence is overwhelming that, when substantial grain growth does occur, it does so over the entire nova sky-i.e. it subtends solid angle $4 \pi$ at the nova. This model also places stringent requirements on the pre-nova evolution of the system: for example, could an Oort-type cloud survive the sequence of events associated with the formation of the white dwarf primary?

\subsection{Summary}

There exists now a distinct prospect of understanding how carbon grains nucleate in nova winds and surely an equivalent understanding of how silicate and silicon carbon grains nucleate can not be far behind. One conclusion is clear. Several authors (e.g. Gallagher 1977; Bode \& Evans 1982; Gehrz 1988) have attempted to relate the grain-forming capabilities of a nova to a single parameter, such as speed class or total ejected mass. However the chemical investigations into grain nucleation, discussed above, show that nucleation may be possible in several regions of a multi-dimensional parameter space. For example, the overabundance of $S$ (ionization potential $10.36 \mathrm{eV}$ ) in nova ejecta has long been known and the abundance of $\mathrm{S}$ may be one of several factors that determine the time for which carbon is shielded from the stellar remnant, and hence the time available for nucleation. It is unlikely therefore that the grain-forming potential of a nova can be assessed on the basis of a single parameter. 


\section{Post-outburst evolution}

Once grains have nucleated growth can occur quite easily and grain growth around novae has been discussed by several authors [see e.g. Gehrz (1988), Bode \& Evans (1989) and references therein]. For our present purposes we shall again consider the evolution of carbon dust.

We should note that the condensation and growth of grains in nova winds will inevitably have a major impact on the evolution of the gaseous ejecta and can not be considered in isolation. As grains form and grow abundances in the ejected material will change substantially. Furthermore, grains will be subject to radiation pressure and will almost certainly be dynamically coupled to the gas. However there seems to have been no detailed study of the effect of grain formation on the evolution of the ejecta; such a study is long overdue.

\subsection{The nature of nova dust}

We first consider whether the carbon dust formed in nova winds is likely to be crystalline (e.g. graphitic) or amorphous. The physical nature of the carbon (or indeed any) condensate is determined by the relative time taken for an atom to migrate over the grain surface $\left(\tau_{m}\right)$ and the time $\left(\tau_{i}\right)$ between the arrival of atoms from the vapour phase onto the grain (see Gail \& Sedlmayr 1984). If $\tau_{m}<\tau_{i}$ then each atom that strikes a grain migrates over the surface until it finds the most favourable site (energetically) to bind to the surface; the result is a crystalline grain. However if $\tau_{m}>\tau_{i}$ the atom settles where it can and an amorphous grain results. Thus whether a carbon grain is crystalline or amorphous is determined by the grain and gas temperature, the activation and binding energies for the material and the supersaturation ratio. It turns out that, during the growth of carbon grains in nova winds, the ratio $\tau_{m} / \tau_{i}$ is such that carbon dust is always amorphous.

\subsection{The 'isothermal' phase}

If the grain emissivity varies with frequency as $\nu^{\alpha}(\alpha \simeq 1)$ the grain temperature $T_{d}$ is expected to decrease monotonically with time $t$. Thus for grains travelling away at constant velocity from a source of constant bolometric luminosity, $T_{d} \propto t^{-2 /(\alpha+4)}$. For fast novae that form dust the dust temperature does indeed decrease in this way.

However during the evolution of the dust shells of moderate speed novae the temperature varies in a complex way, first reaching a minimum, then rising before finally going into decline; this behaviour is sometimes referred to as the 'isothermal' phase. For a grain of radius $a, T_{d} \propto a^{-1 /(\alpha+4)}$. The rise in temperature seen during the 'isothermal' phase can therefore be understood in terms of grain destruction, and chemisputtering of carbon dust by protons has been suggested as the destructive agent (Mitchell \& Evans 1984; Mitchell et al. 1986). Indeed these authors predicted the presence of 3.4 and $11 \mu \mathrm{m}$ emission features-arising from $\mathrm{C}-\mathrm{H}$ bond resonances-during the grain destruction phase. However the increase in grain temperature may simply be due to the fact that, when the hydrogen is completely ionized, grains are exposed to an additional source of short wavelength radiation (Lyman continuum photons) following the opening of the 'Lyman window'. Indeed the fact that the 'grain destruction' time coincides with the hydrogen ionization time was noted by Mitchell \& Evans (1984).

\section{3 'PAH' emission in novae}

Without doubt one of the most exciting developments of late has been the infrared behaviour of V842 Cen (Nova Cen 1986). The early infrared spectroscopic development of this moderate speed nova showed, in addition to atomic and ionic lines, the first overtone vibrational-rotational 
transition of CO at $2.3 \mu \mathrm{m}$ (Hyland \& McGregor 1989). As noted by Rawlings \& Williams (1989) the appearance of $\mathrm{CO}$ is a prerequisite for grain formation and indeed, in common with may other novae of this speed class, V842 Cen developed an optically thick dust shell after $\sim 50$ days.

Hyland \& McGregor (1989) reported the appearance of broad emission features between 3.2 and $3.6 \mu \mathrm{m}$ in V842 Cen some 300 days after outburst. The features are reminiscent of the features commonly associated in other sources with emission by polycyclic aromatic hydrocarbons (PAHs), although the details differ somewhat. Smith et al. (1989) have reported that the dust in this nova shows the characteristic silicate feature at $9.7 \mu \mathrm{m}$, as well as a PAH feature at $11.3 \mu \mathrm{m}$. The apparent presence of both silicate (i.e. $\mathrm{O}>\mathrm{C}$ ) and carbon-based $(\mathrm{C}>\mathrm{O})$ dust is not of course unheard of in circumstellar environments (e.g. Lloyd-Evans 1985; Little-Marenin 1986). However in isolated stars this can be understood in terms of mass-ejection during a previous evolutionary phase, when the chemical make-up of the star was completely different.

In the case of nova dust the situation is not as straightforward because the different grains are condensing in the ejecta of a single eruption. The variety of the dust may point to the fact that substantial depletion of either $\mathrm{O}$ or $\mathrm{C}$ (probably the latter) is occurring so that the balance of abundances in the ejecta tips from the carbon-rich to the oxygen-rich case; thus carbon dust may form early in the eruption while oxygen-rich dust condenses later on. On the other hand there may well be significant abundance gradients in the ejected material, which may suggest that the initial TNR event might have been fairly localized. However in novae known (from spectroscopy) to be extremely carbon-rich, the apparent condensation of materials having both carbon-rich and oxygen-rich characteristics may point to the latter alternative.

The infrared emission properties of PAHs have been fully discussed by Allamandola (1989) and coworkers (Allamandola et al. 1989), who demonstrate that the relative strengths of the various infrared emission features carry a great deal of information about the nature of the emitting species. Thus the relative strength of the 3.28 and $11.3 \mu \mathrm{m}$ features is dependent on the number of carbon atoms in the emitting PAH. As always, simultaneous observations in the two wavebands are needed to draw any significant conclusion but it seems that the $11.3 \mu \mathrm{m}$ feature in V842 Cen was very weak by comparison with the $3.28 \mu \mathrm{m}$ feature; if this is the case it points to PAHs with fewer than $\sim 10$ carbons. Whereas the $3.28 \mu \mathrm{m}$ feature is typically found in aromatics, features in the 3.4-3.6 $\mu \mathrm{m}$ range tend to be associated with saturated hydrocarbons. In V842 Cen the $3.28 \mu \mathrm{m}$ feature was weaker, relative to those at 3.4-3.6 $\mu \mathrm{m}$, compared with the situation in other sources in which these features appear (Hyland \& McGregor 1989). The relative strengths of these features in V842 Cen suggests that the ratio of saturated to unsaturated hydrocarbons was somewhat different to that normally encountered.

It should be pointed out that PAH emission at 3.28 and $11.3 \mu \mathrm{m}$ is accompanied by a very strong broad feature at $7.7 \mu \mathrm{m}$; obviously observations at this wavelength, concurrent with any in the 3 and $8-13 \mu \mathrm{m}$ windows, of future V842 Cen-type novae is highly desirable. Also, the possibility of broad emission in the red, such as the 'extended red emission' (ERE) seen around 6500-7500 $\AA$ in the 'Red Rectangle' (Schmidt et al. 1980) and various other objects, should also be borne in mind. Since optical spectroscopy of novac is more routinely carried out than infrared spectroscopy ERE would be comparatively easy to look for.

Mitchell et al. (1986) predicted that, during the optically thick phase of nova dust shell development, chemisputtering of carbon dust by hydrogen would occur, giving rise to emission by $\mathrm{CH}$ groups at $\sim 3.3 \mu \mathrm{m}$ and $11.3 \mu \mathrm{m}$, particularly during deep minimum. However infrared spectra of V842 Cen obtained by Hyland \& McGregor (1989) in 1987 January-when the light curve was well into its deep minimum-show that the dust continuum was smooth in the $3 \mu \mathrm{m}$ region, and the $11.3 \mu \mathrm{m}$ PAH feature was not present in a spectrum obtained by Smith et al. (1989) 162 days after outburst, when the nova was recovering from its minimum. It was not until the light curve 
had fully recovered that later spectra (Hyland \& McGregor 1989; Smith et al. 1989) revealed the $\mathrm{PAH}$ features in the $3 \mu \mathrm{m}$ and $11 \mu \mathrm{m}$ windows.

While it is tempting to associate the 3 and $11 \mu \mathrm{m}$ emission features seen in V842 Cen with the process suggested by Mitchell et al. (1986), we have already noted that grain destruction is not necessarily required to account for the 'isothermal' phase. However the process discussed by Mitchell \& Evans (1984) may be relevant in this respect. Carbon grains in nova winds are (as noted above) amorphous and indeed Allamandola et al. (1989) have discussed the relationship between PAHs and amorphous carbon, and the growth of the latter from the former. Carbon grains are expected to be negatively charged and such grains will grow rapidly while carbon is ionized. When the hydrogen is fully ionized however we can expect $\mathrm{H}$ to become attached to grain surfaces; the flood of Lyman continuum photons that follows the opening of the Lyman window would then be sufficient to excite emission by the $\mathrm{C}-\mathrm{H}$ bonds on the grain surface.

\section{Post-nova evolution}

The fine-grained matrix component of carbonaceous meteorites suffered very little (thermally) during the formation of the solar system. Fortunately the ultimate origin of grains (e.g. carbon stars, red giant stars etc.) is potentially identifiable because each of these sources of dust uniquely 'labels' the grains isotopically and novae are no exception. The possibility that nova dust may be accessible for laboratory analysis was noted by Clayton \& Hoyle (1976), although it is now clear that the contribution of novae to the interstellar dust population is negligible. However laboratory work on carbonaceous meteorites suggests that these could well contain material that has isotopic signatures characteristic of TNR in novae and which cannot be explained in terms of subsequent processing in interstellar space or in the solar system.

'Type 2' silicon carbide in carbonaceous meteorites (also known as ' $\mathrm{Ce} \mathrm{SiC}$ ') contains ${ }^{22} \mathrm{Ne}$, with little or no other noble gases. It seems that this isotope arises from the $\beta^{+}$decay of ${ }^{22} \mathrm{Na}$, which is locked up in the SiC. The short lifetime $(2.6 \mathrm{yr})$ of ${ }^{22} \mathrm{Na}$ requires that it be produced and locked up in the silicon carbide on a very short timescale, such as occurs in a nova outburst (Anders et al. 1989); the possibility that ${ }^{22} \mathrm{Na}$ is confined in nova dust as a metallofullerene was suggested by Kroto (1988). It may also be worth noting that most of the mass of Type $2 \mathrm{SiC}$ is in the $0.1-1 \mu \mathrm{m}$ size range, similar to that expected for nova dust, although too much significance should perhaps not be attached to this fact.

Tang et al. (1989) have looked at the relationship between ${ }^{14} \mathrm{~N} /{ }^{15} \mathrm{~N}$ and ${ }^{12} \mathrm{C} /{ }^{13} \mathrm{C}$ in carbonaceous chondrite meteorites and they found substantial evidence for isotopic ratios that are determined by explosive nucleosynthesis, such as that occurring in novae. It may be worth pointing out that, if this is the case, meteorites are potentially a source of information about the chemical evolution of nova systems. The dust that is locked up in meteorites was produced over $5 \times 10^{9}$ years ago and any nova dust in meteorites could provide information about TNR and abundances in novae during the early evolution of the Galaxy.

\section{Concluding remarks}

We finally mention four areas in which further work is needed to further our understanding of dust formation and evolution in classical novae:

- Despite the fact that infrared observations of novae are becoming increasingly commonplace and more detailed, polarimetry of novae during the dust (or any) phase remains lacking. This is extremely unfortunate because the wavelength-dependence of polarization is a powerful means of determining grain size and indeed, spectropolarimetry with reasonable time 
resolution could give the grain growth (and where appropriate, destruction) rate. This would provide an invaluable handle on grain growth (and destruction) processes.

- Spectroscopy in the extreme red and in the $7 \mu \mathrm{m}$ band are desirable to complement spectroscopy in the 3 and $11 \mu \mathrm{m}$ windows.

- While it now possible to understand how carbon grains nucleate in nova winds, further work needs to be done in understanding how and why some novae produce a variety of dust types. It may be that different species condense at different times, or that there are abundance gradients in the ejecta. An explanation for the latter may have to come from refinement of TNR models, while the former may be related to the following item.

- Little has been done on the effect of radiation pressure on the growing grains, on the way in which they are coupled to the surrounding gas, and on the depletion of species in the gas phase as grains grow. As noted above the formation of grains must have a major impact on the evolution of the gaseous ejecta.

\section{References}

Allamandola, L. J., 1989. In IAU Symposium 135 Interstellar Dust, in press.

Allamandola, L. J., Tielens, A. G. G. M. \& Barker, J. R., 1989. Astrophys. J. Suppl., in press.

Anders, E., Lewis, R. S., Tang, M. \& Zinner, E., 1989. In IAU Symposium 135 Interstellar Dust, in press.

Bode, M. F. \& Evans, A., 1980. Astron. Astrophys., 89, 158.

Bode, M. F. \& Evans, A., 1982. Mon. Not. Roy. Astron. Soc., 200, 175.

Bode, M. F. \& Evans, A., 1983a. Mon: Not. Roy. Astron. Soc., 203, 285.

Bode, M. F. \& Evans, A., 1983b. Quart. J. Roy. Astron. Soc., 24, 83.

Bode, M. F. \& Evans, A., 1989. Chapter 9 of Classical Novae, J. Wiley, Chichester.

Bode, M. F., Evans, A., Whittet, D. C. B., Aitken, D. K., Roche, P. F. \& Whitmore, B., Mon. Not. Roy. Astron. Soc., 207, 897.

Callus, C. M., Albinson J. S., \& Evans, A., 1987. Astrophys. Sp. Sci., 131, 437.

Carmer, C. S. \& Frenklach, M., 1989. Applied Physics Letters, 54, 1430.

Clayton, D. D. \& Hoyle, F., 1976. Astrophys. J., 203, 490.

Curl, R. F. \& Smalley, R. E., 1988. Science, 242, 1017.

Donn, B., Wickramasinghe, N. C., Hudson, J. P. \& Stecher, T. P., 1968. Astrophys. J., 153, 451.

Draine, B. T.,1979. Astrophys. Space Sci., 65, 313.

Duley, W. W. \& Williams, D. A., 1984. Interstellar Chemistry. Academic Oxford.

Frenklach, M., Carmer, C. S. \& Feigelson, E. D., 1989. Nature, 339, 196.

Gail, H.-P. \& Sedlmayr, E., 1984. Astron. Astrophys., 132, 163.

Gallagher, J. S., 1977. Astron. J., 82, 209.

Gehrz, R. D., 1988. Ann. Rev. Astron. Astrophys., 26, 377.

Gehrz, R. D., 1989. These proceedings.

Gehrz, R. D., Ney, E. P., Grasdalen, G. L., Hackwell, J. A. \& Thronson, H. A., 1984. Astrophys. $J ., 281,303$.

Gehrz, R. D., Harrison, T. E., Ney, E. P., Mathews, K., Neugebauer, G., Elias, J., Grasdalen, G. L. \& Hackwell, J. A., 1988. Astrophys. J., 329, 894.

Geisel, S. L., Kleinmann, D. E. \& Low, F. J., 1970. Astrophys J., 161, L101.

Goebel, J. H., 1986. Bull. Amer. Astron. Soc., 18, 1003.

Hyland, A. R. \& McGregor, P. J., 1989. In IAU Symposium 135 Interstellar Dust, in press.

Jenkins, R. N. \& Evans, A., 1988. In Dust in the Universe, p.373, eds M. E. Bailey \& D. A. Williams, Cambridge University Press, Cambridge. 
Johnson, D., Friedlander, M. W. \& Katz, J. I., 1987. Bull. Amer. Astron. Soc., 19, 724.

Kroto, H., 1988. Science, 242, 1139.

Lewis, J. S. \& Ney, E. P., 1979. Astrophys. J., 234, 154.

Little-Marenin, I. R., 1986. Astrophys. J., 307, L15.

Lloyd-Evans, T., 1985. Mon. Not. Roy. Astron. Soc., 217, 493.

McLaughlin, D. B., 1935. Publ. Amer. Astron. Soc., 8, 145.

Matese, J. J., Whitmire, D. P. \& Reynolds, R. T., 1989. In IAU Symposium 135 Interstellar Dust, in press.

Mitchell, R. M. \& Evans, A., 1984. Mon. Not. Roy. Astron. Soc., 209, 945.

Mitchell, R. M., Evans, A. \& Albinson, J. S., 1986. Mon. Not. Roy. Astron. Soc., 221, 663.

Mitchell, R. M., Evans, A. \& Bode, M. F., 1983. Mon. Not. Roy. Astron. Soc., 205, 1141.

Rawlings, J. M. C., 1988. Mon. Not. Roy. Astron. Soc., 232, 507.

Rawlings, J. M. C. \& Williams, D. A., 1989. Mon. Not. Roy. Astron. Soc., in press. See also these proceedings.

Roche, P. F., Aitken, D. A. \& Whitmore, B., 1984. Mon. Not. Roy. Astron. Soc., $211,531$.

Schmidt, G. D., Cohen, M. \& Margon, B., 1980. Astrophys. J., 239, L133.

Shara, M. M., Livio, M., Moffat, A. F. J. \& Orio, M., 1986. Astrophys. J., 311, 163.

Smith, C. H., Aitken, D. K. \& Roche, P. F., 1989. These proceedings.

Snijders, M. A. J., Batt, T. J., Seaton, M. J., Blades, J. C. \& Morton, D. C., 1984. Mon. Not. Roy. Astron. Soc., 211, 7P.

Snijders, M. A. J., Batt, T. J., Roche, P. F., Seaton, M. J., Morton, D. C., Spoelstra, T. A. T. \& Blades, J. C., 1986. Mon. Not. Roy. Astron. Soc., 228, 329.

Tang, M., Anders, E., Hoppe, P. \& Zinner, E., 1989. Nature, 339, 351. 